\title{
Effects of Bursty Event Traffic on Synchrophasor Delays in IEEE C37.118, IEC61850, and IEC60870
}

\author{
Yiming Wu, Lars Nordström \\ Industrial Information and Control Systems \\ KTH-Royal Institute of Technology \\ Stockholm, Sweden 10044 \\ Email: yimingw@kth.se
}

\author{
David E. Bakken \\ The School of Electrical Engineering and Computer Science \\ Washington State University \\ Pullman, Washington, USA
}

\begin{abstract}
Electric grids are increasingly relying on communication as grids get more stressed due to increasing penetration of renewables, inadequate transmission growth, and other factors. The IEEE detailed power system models have been widely used in power research. But lack of communication topologies or requirements leads to difficulties for results comparison or guidelines for utilities. Further, power grid communication often meet latency requirement by application during the steady state since in such state light traffic are generated. However, identical requirements can be stringent in the bulk power system over hundreds of miles especially when bursty aperiodic event messages are generated during a power disturbance. To address these shortcomings, this paper presents a detailed model of realistic communication for IEEE-14 bus system. Simulation measurement shows significant effects of bursty traffic from GOOSE disturbance event message over Wide Area Network on the delays suffered by other periodic traffic such as synchrophasor data, SCADA polling data, and Sampled Value exchanged between substations. The results also reveals significant scalability issues of centralized control center architecture.
\end{abstract}

Index Terms-Bursty Event Traffic, Quality of Service, WAMC, IEC61850, C37.118.

\section{INTRODUCTION}

Electric grids are increasingly relying on communications as grids get more stressed due to increasing penetration of renewables, inadequate transmission growth, and a myriad of other factors. The IEEE provides very detailed power system models that are widely used in power research papers. However, these models do not include any communications topologies or requirements. As a result of this, and that power researchers in general are not network protocol, middleware, or real-time computing experts, power researchers almost always ignore the effect of communications anomalies on power applications.

Further, power grid communications often meet application delay requirements during the (relatively calm and predictable) steady state. Such requirements can be quite stringent in the bulk power system over many hundreds of miles [1]. During a power disturbance many (aperiodic) event messages (report by exception alarms) may be sent over the same network as the synchrophasor data. The effect of these event messages on application performance has been noted elsewhere [2]. But, to the best of our knowledge, it has not been studied. A semantic-based intelligent alarming is raised in order to avoid sending all alarms/events to the control center as present state of the practice. However, it is not yet readily available. Communications-related standards for the power grid have different mechanisms and properties. Unfortunately, little guidance is given for their configuration and its effect on communications performance, and are typically written by power engineers, not network protocol engineers. For example, The IEEE C37.118 standard for synchrophasor data supports both TCP and UDP. The IEC 61850 substation automation communications were originally designed directly on top of Ethernet frames. The IEC60870-5-104 defines communication from Remote Terminal Unit (RTU) to Supervisory Control and Data Acquisition (SCADA). Our research is addressing the above shortcomings. The contributions of this paper are:

- A detailed model of realistic communications combined with the standard IEEE 14-bus model

- Simulation measurements that show significant effects of bursty traffic from tunneling GOOSE (IEC61850-82) disturbance event messages over Wide Area Network (WAN) on the delays suffered by steady state periodic traffic, using both TCP and UDP for IEEE C37.118 synchrophasor traffic and using Sampled Value over UDP for IEC61850-90-2

- Communication requirements for different applications, including State Estimation, Optimal Power Flow, Remedial Action Scheme, Power Oscillation Damping, Current Differential Protection in the 14-bus model

Our conclusions include:

- The centralized control center architecture has significant scalability issues

- 10 Mbit data lines are very inadequate in the face of such bursty event traffic, but 100 Mbit speeds suffice

- Without any strong QoS mechanisms such as with GridStat [1] or ATM, the only way to ensure adequate performance is ensuring low link utilization (i.e., massive overprovisioning of bandwidth)

The remainder of this paper is organized as follows. Section II overviews widely used standards in present power systems and communication patterns. Section III describes the methodology of creating a model of realistic communication for standard IEEE 14-bus model. In order to determine data 
links and their Quality of Service (QoS) requirements in this model, as described in Section IV, a review of power system application literature has been performed. In section V, scenarios using different synchrophasor data implementation and different bandwidth parameters are described and demonstrated. Based on obtained result, conclusion is drawn and future research is presented in the Section VI.

\section{Grid COMmunication PATterns}

By reviewing widely used power industrial standards (i.e. IEC61850, IEEE C37.118, and IEC60870), the communication patterns can be divided into four different types: periodic, event based aperiodic, event based periodic, and polling.

- Periodic communication means a data source updates its measurement or status periodically. Phasor Measurement Unit (PMU) and Merging Unit (MU) are typical devices implementing periodical communication pattern.

- Event based aperiodic communication means the data is sent due to an event, such event can be operator request or power system event. For example, the substation monitoring video stream and data retrieving for post disturbance analysis apply the event based aperiodic communication normally.

- Event based periodic communication combines event based aperiodic and periodic communication. It has two different states: normal and event. In normal state, data source sends messages using long interval time. However, once event occurs, data source immediately sends updated messages using short interval time try to ensure that the receiver obtains the updated information. Generic Object Oriented Substation Events (GOOSE) and Manufacture Messaging Specification (MMS) Report use this communication pattern.

- Polling is widely used in SCADA systems. Control Center queries data from RTUs in each substation one by one to obtain the latest measurement and status.

These different communication patterns have been modeled in a communication simulator and the typical traffic patterns are illustrated in following figures. Since event based communication is normally driven by the operator. It also includes report by exception when server values cross thresholds or change sufficiently. Consequently, QoS requirements of such communication pattern is the lowest among these communication patterns due to human involved decision making is slower than automatic control process. Therefore, in this paper, event based communication patterns are not included.

In the upper part of Figure1, traffic characteristic of PMU is given. The report rate of the PMU is set as 60 packets per second [3]. Similarly, traffic characteristic of MU is given in the lower part of Figure1. According to Light Edition of IEC61850-9-2, report rate of MU is set as 4000 packets per second [4].

GOOSE is modeled as shown in Figure2. In the steady state, Intelligent Electronic Devices (IED) send GOOSE messages 1 packet per second. However, once the event occurs, the standard allows IEDs send 1000 packets per second in the

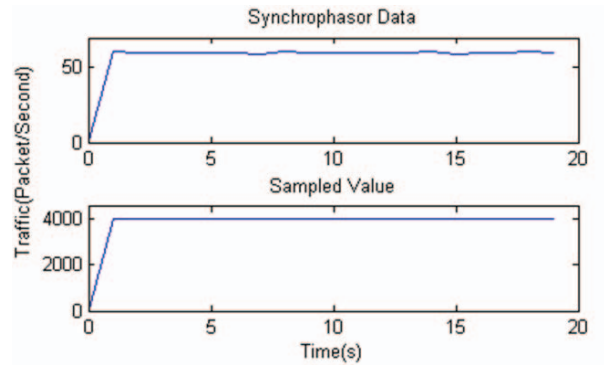

Fig. 1: Periodic communication pattern.

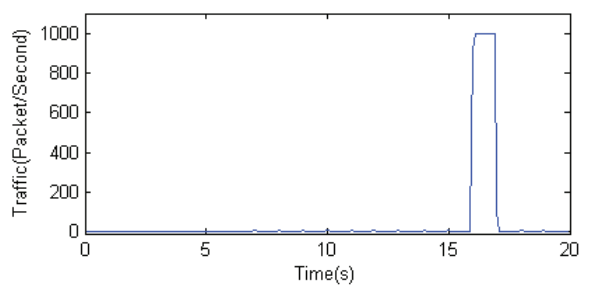

Fig. 2: GOOSE communication pattern.

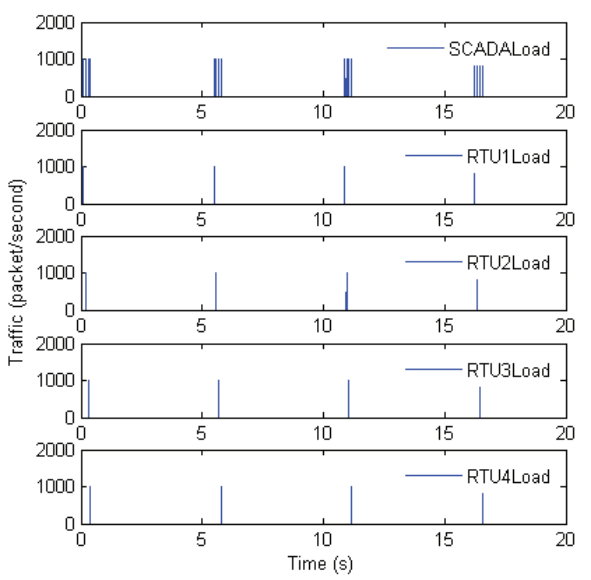

Fig. 3: SCADA communication pattern.

following one second to ensure the receiver(s) getting the updated information on time.

Finally, SCADA communication pattern is given as shown in Figure3. The SCADA system queries RTUs one by one. Each RTU replies with its latest information to the SCADA system after receiving the query message. Normally, SCADA system takes up to several seconds for a round of polling [5].

\section{Power Models And Their Supporting COMMUNICATION MODELS}

IEEE Power System Models (i.e. IEEE 14-bus system, IEEE 30-bus system, IEEE 57-bus system, IEEE 118-bus system, and IEEE 300-bus system) have detailed parameters for power equipments such as transmission lines, loads, generators, and topology of the system [6]. However, none of them includes supporting communication model outside of the substation scope. Although research on communication for power system 
is a hot topic, there is lack of communication infrastructure modelling parameters. Consequently, it is difficult to reproduce studied models or to compare results from different projects. In addition, there is no guideline for utility to follow. To avoid such weakness, the methodology to model power system communication infrastructure in a realistic way is illustrated in this section.

\section{A. Supporting Communication Modelling Methodology}

To model the communication infrastructure of a power system, the assumption for topology is given as following: A communication link is assumed between two substations if two substations are connected by one (or more) transmission line(s). In power system, transmission lines are protected by substations located at its two ends. Normally, current differential protection is implemented in these two substations to protect the transmission line from faults [7]. To enable current differential protection, a fiber optic link dedicated only to traffic between the two endpoint devices is used. In this paper, it is assumed each substation is equipped with a router to connect substation Local Area Network (LAN) to power system WAN. The WAN is built by connecting these substation routers together by fiber optic cables between substations. In this case these fiber optic cables are shared by different application data links instead of being dedicated to current differential protection. Therefore, a realistic baseline topology of communication network can be obtained as same as topology of power system transmission lines. The fiber optical cable length can also be obtained by Equation 1

$$
I_{i j}=R_{i j} / \hat{R}
$$

where $R_{i j}$ is transmission line resistance between bus $i$ and bus $j . \hat{R}$ is typical overhead transmission line resistance in p.u. $/ \mathrm{km}$. It can be obtained by Equation 2

$$
\hat{R}=\frac{\hat{R}_{\text {typical }}}{R_{\text {base }}}=\frac{\hat{R}_{\text {typical }} \times S_{\text {base }}}{V_{\text {base }}^{2}}=0.0001323 p . u . / \mathrm{km}
$$

where $\hat{R}_{\text {typical }}=0.05 \Omega / \mathrm{km}, S_{\text {base }}=100 \mathrm{MW}$ is base apparent power and $V_{\text {base }}=230 \mathrm{kV}$ is base voltage. Therefore, the transmission line length can be calculated.

\section{B. Communication Infrastructure Modeling for IEEE 14-bus system}

In this paper, a modified IEEE 14-bus system is studied as shown in Figure4. In this system, an HVDC link is added between Bus5 and Bus9 providing Power Oscillation Damping control in order to solve the challenges of integration of renewable sources [8].

Based on the assumption and methodology described in previous part, the supporting communication infrastructure topology can be obtained. Although there are two transmission line between Bus1 and Bus2, only one communication link is provided between these two buses. Since there is no transmission line between Bus5 and Bus6, these two buses

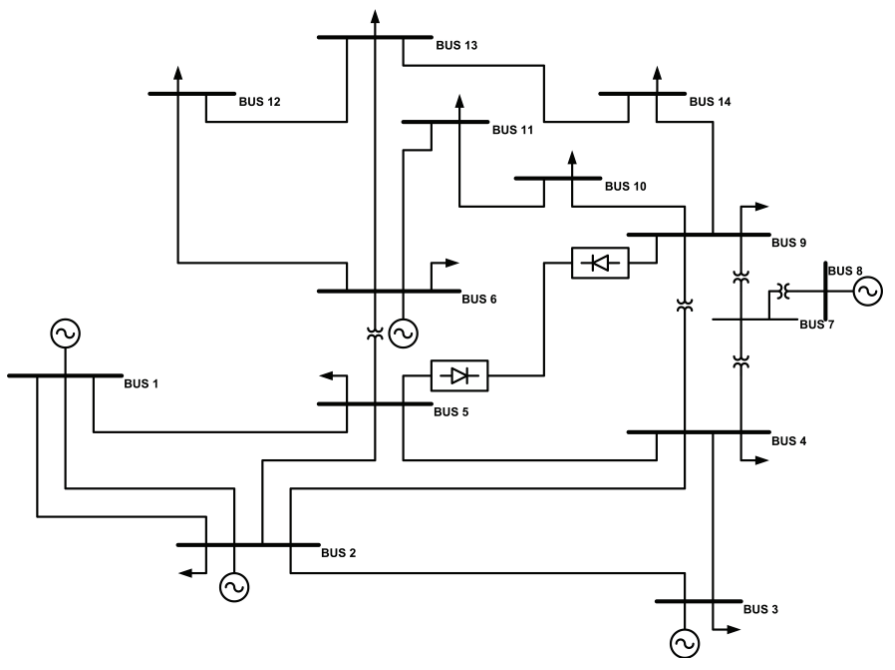

Fig. 4: IEEE 14-bus System.

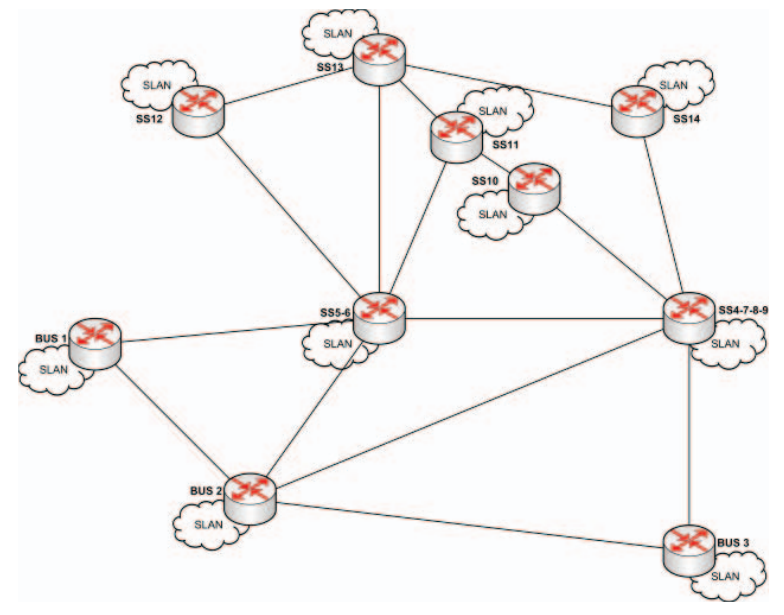

Fig. 5: IEEE 14-bus Communication Infrastructure Topology.

are considered in the same substation $S S_{5 \_}$. Similarly, Bus4, Bus7, Bus8, and Bus9 are considered in the same substation $S S_{4 \_7 \_8 \_9}$. Each of the remaining buses are considered as one substation $S S_{i}$ where $i$ is the corresponding bus number. It is assumed the Control Center of the system is located near to $S S_{5 \_}$. Control Center accesses power system WAN through substation router located at $S S_{5 \_}$. Therefore, the supporting communication infrastructure of IEEE 14-bus system can be obtained and described as Figure 5.

The length parameter of communication link for the study system is given in Table I.

\section{Overview of Applications Studied}

In this section, applications in related to IEEE 14-bus system are determined by review of literature. The data link configuration and QoS requirements are presented as a reference for result discussion.

\section{A. Applications and Their QoS Requirements}

The applications deployed in this paper is based on literature review. There might be more applications can be implemented 
TABLE I: Communication Physical Link Parameters

\begin{tabular}{|c|c|c|c|}
\hline Link & End Node A & End Node B & Length $(\mathrm{km})$ \\
\hline$L_{1}$ & $S S_{1}$ & $S S_{2}$ & 146.46 \\
\hline$L_{2}$ & $S S_{1}$ & $S S_{5 \_6}$ & 408.31 \\
\hline$L_{3}$ & $S S_{2}$ & $S S_{3}$ & 355.11 \\
\hline$L_{4}$ & $S S_{2}$ & $S S_{4} \_7 \_8 \_9$ & 439.15 \\
\hline$L_{5}$ & $S S_{2}$ & $S S_{5 \_}$ & 439.15 \\
\hline$L_{6}$ & $S S_{3}$ & $S S_{4}$ & 506.40 \\
\hline$L_{7}$ & $S S_{4 \_7 \_8 \_9}$ & $S S_{5 \_6}$ & 100.89 \\
\hline$L_{8}$ & $S S_{5 \_} 6$ & $S S_{11}$ & 717.78 \\
\hline$L_{9}$ & $S S_{5 \_6}$ & $S S_{12}$ & 928.85 \\
\hline$L_{10}$ & $S S_{5 \_} 6$ & $S S_{13}$ & 499.91 \\
\hline$L_{11}$ & $S S_{4 \_7 \_8 \_9}$ & $S S_{10}$ & 240.39 \\
\hline$L_{12}$ & $S S_{4 \_7 \_8 \_9}$ & $S S_{14}$ & 960.59 \\
\hline$L_{13}$ & $S S_{10}$ & $S S_{11}$ & 620.06 \\
\hline$L_{14}$ & $S S_{12}$ & $S S_{13}$ & 1669.53 \\
\hline$L_{15}$ & $S S_{13}$ & $S S_{14}$ & 1291.73 \\
\hline$L_{16}$ & $S S_{5 \_6}$ & $C C$ & 10 \\
\hline
\end{tabular}

in the studied power system. However, for demonstration purpose, only following applications are considered in this paper.

- Control Center Applications

- Topology Processing (TP)

- Online State Estimation (SE)

- Optimal Power Flow (OPF)

- Voltage Stability Analysis

- Secondary Frequency Control

- Secondary Voltage Control

- Remedial Action Scheme (RAS)

In conventional power system architecture, system wide control applications are almost all located in control center. The control center deploys a SCADA system to obtain system measurement data from each substation. Based on the breaker and switch status, power system topology can be determined by TP application. Based on result of TP, SE can be performed to obtain states such as power flows, voltage levels, and phasors of the system. These states are used for OPF, Voltage Statbility Analysis, Secondary Frequency Control, and Secondary Voltage Control. Recently, Remedial Action Scheme (RAS) is another hot topic focuses on system wide protection. In this paper, only TP, online SE, and RAS is considered since the rest control center applications relying on result of TP and SE.

- Substation Control Applications

- Primary Frequency Control

- Primary Voltage Control

- Power Oscillation Damping

- Power System Stabilizer

In Substation, Primary Frequency Control, Primary Voltage Control, Power Oscillation Damping (POD), Power System Stabilizer (PSS) are deployed. Normally Primary Frequency Control, Primary Voltage Control, and PSS use measurements from their local substations. Some research has investigated using global signals for POD to get better control performance [9] [10]. Therefore, in this paper, only POD is studied.

- Substation Protection Applications
- Current Differential Protection [7]

- Interlocking [7]

To protect the power system equipment such as transformer, generator, transmission line bus bar, feeder, and so on from faults. Protection applications are implemented in IEDs. Most of these protection applications, for instance generator protection, transformer protection, bus bar protection, and feeder protection, use measurement from their local substations. However, transmission line protection requires measurement data from the substation at the other end of the transmission line. To ensure the selectivity of protection functions, interlocking is applied to use the tripping information from other substations to block unnecessary trips. In this paper, only current differential protection application for transmission line and interlocking application are considered due to their requirements of WAN communication.

The summary of applications and their corresponding QoS Requirements are listed in Table II. Protection applications (i.e. current differential line protection and interlocking) have most restrict requirements on QoS. Control applications such as RAS and POD has less latency and report rate requirements than protection applications but higher than monitoring applications (i.e. TP and Online SE) [11]. These requirements are used as reference for the result part in this paper. More general communication requirements for the bulk power system can be found in [1] and [12].

TABLE II: QoS Requirements of Applications

\begin{tabular}{|l|l|l|}
\hline Application & Report Rate(pkts/s) & Latency(s) \\
\hline Topology Processing & $>0.2$ & $<5.000$ \\
\hline Online State Estimation & $>0.2$ & $<5.000$ \\
\hline Remedial Action Scheme & Capture Event & $<0.200$ \\
\hline Power Oscillation Damping & $>10$ & $<0.250$ \\
\hline Current Differential Line Protection & $>720$ & $<0.005$ \\
\hline Interlocking & Capture Event & $<0.005$ \\
\hline
\end{tabular}

\section{B. Data Link Configuration for Applications}

In this part, data link configuration for each application is determined based on the relevant literature review, as summarized in Table III.

The TP requires status of breakers and switches in each substation [13] [14] [15]. The Control Center SCADA polling RTU to update these information using IEC60870-5-104 protocol as shown in the 1st row in Table III [16]. RTUs in each substation reply their latest data to SCADA as shown in 2nd row in Table III. A two stage state estimation is deployed in this system. It uses the power system real time topology, output from TP, and conventional state estimation measurements configured in RTUs response such as active power and reactive power from PQ buses, voltage and their phasor angle from slack bus, and active power and voltage from PV buses as shwon in 1st and 2nd row in Table III. In addition, it also requires synchrophasor data from $S S_{2}$, $S S_{4_{-} 7_{-} 8_{-} 9}$, and $S S_{5 \_6}$ as shown in 11st row in Table III. A Centralized RAS (CRAS) proposed in [17] is chosen for power system protection in wide area. According to [17], 
TABLE III: Data Link Configuration

\begin{tabular}{|l|l|l|l|}
\hline Send Node & Receive Node & Protocol & Packet Size(Bytes) \\
\hline$C C_{S C A D A}$ & $S S_{i} R T U$ & IEC60870 & 100 \\
\hline$S S_{i} R T U$ & $C C_{S C A D A}$ & IEC60870 & 200 \\
\hline$S S_{a} I E D$ & $C C_{S C A D A}$ & GOOSE over UDP & 1630 \\
\hline$S S_{b} I E D$ & $C C_{S C A D A}$ & GOOSE over UDP & 2534 \\
\hline$S S_{c} I E D$ & $C C_{S C A D A}$ & GOOSE over UDP & 1178 \\
\hline$S S_{d} I E D$ & $C C_{S C A D A}$ & GOOSE over UDP & 274 \\
\hline$S S_{1} M U$ & $S S_{2} I E D$ & GOOSE over UDP & 922 \\
\hline$S S_{2} I E D$ & $S S_{1} I E D$ & GOOSE over UDP & 922 \\
\hline$S S_{m} M U$ & $S S_{n} I E D$ & SV over UDP & 162 \\
\hline$S S_{m} I E D$ & $S S_{n} I E D$ & GOOSE over UDP & 274 \\
\hline$S S_{j} P M U_{x}$ & $C C_{P D C}$ & C37.118 & 72 \\
\hline$S S_{k} P M U_{y}$ & $S 5_{P D C}$ & C37.118 & 72 \\
\hline
\end{tabular}

Node names in this table is $<$ Substation $><$ Equipment $>$, for example, $S S 1 I E D$ is IED in substation 1 and $S S 2 P M U$ is PMU in substation2. $C C_{S C A D A}$ is control center SCADA, $C C_{P D C}$ is Control Center Phasor Data Concentrator(PDC) and $S S 5_{P D C}$ is PDC in substation 5;

$i=1,2,3,4 \_7 \_8 \_9,5 \_6,10,11,12,13,14$;

$a=1,4 \_7 \_8 \_9,5 \_6,13 ; b=2$;

$c=3,4 \_7 \_8 \_9,10,11,12,14 ; d=4 \_7 \_8 \_9$;

$m$ and $n$ come as a pair means Substation $m$ and Substation $n$ is connected by a transmission line;

$(j, x)=(2,\{1,2,3,4,5\}),(6,\{1,2,3,4\}),(8,1),\left(4 \_7 \_8 \_9,\{1,2,3\}\right)$ $(k, y)=(1,1),(2,1),(3,1),\left(4 \_7 \_8 \_9,1,2,3\right),(14,1)$

CRAS implemented in control center requires circuit breaker status, voltage measurement, and rate of change of analogue measurements from the substations. In order to capture the real time information, GOOSE tunneling technique is used to carry time sensitive event messages [7]. Therefore, data links shown in the 3rd to 6th rows in the Table III are configured. Since there is one HVDC link implemented in the system, this HVDC link is used to control power oscillations in the system. A Model Predictive Control algorithm is proposed in [8] for power oscillation damping. Such algorithm is implemented in the system. The input signals for POD controller is voltage magnitudes and their phasors from $S S_{1}, S S_{2}, S S_{3}, S S_{4 \_7 \_} \_9$, $S S_{5 \_6}$, and $S S_{14}$ as shown in the last row in the Table III. The protection functions, Current Differential Line Protectiona (CDLP) and Interlocking, use GOOSE and SV over UDP tunneling to allow measurement and breaker status information exchange between two substations. To ensure the CDLP algorithm working properly, three phase current measurement of a transmission line is shared between substations at both end of the line [7]. Substations at both end of transmission line exchange trip commands each other to block unnecessary trip (i.e. overcurrent protection) when a fault occurs or ensure the safety of maintenance [7]. The data link configuration for CDLP and interlocking are shown as 7th to 10th rows in the Table III.

The packet size of each data link is determined as follows and results are listed in the Table III:

- Synchrophasor Data: fixed 72 bytes

- SCADA polling data:

- Request: 100 bytes

- Response: 200 bytes

- SV over UDP tunneling: fixed 162 bytes
- GOOSE over UDP tunneling: determined by Equation 3

$$
L=L_{h}+i \times L_{\text {analog }}+j \times L_{\text {bool }}+(i+j) \times L_{q}+L_{t}
$$

where $L$ is packet length, $L_{h}$ is length of GOOSE message headers, $i$ is the number of analog signal in the GOOSE message, $L_{\text {analog }}=6$ bytes is length of each analog signal, $j$ is number of boolean signal in the GOOSE message, $L_{\text {bool }}=3$ bytes is length of each boolean signal, $L_{q}=5$ bytes is length of quality data of each signal in the GOOSE message, $L_{t}=10 b y t e s$ is the length of time stamp in the message.

\section{Simulation Results}

The simulation is carried out in the OPNET ${ }^{\mathrm{TM}}$ communication simulator. By performing simulation using different scenarios, results are obtained in this section. First, different simulation scenarios are described. Then results are presented.

\section{A. Scenarios}

By combination of different link bandwidth and synchropha):sor data configuration, four scenarios have been performed for simulation. Two link bandwidth are considered: $10 \mathrm{mbps}$ and $100 \mathrm{mbps}$. Two different synchrophasor data configuration are considered: UDP and TCP. The results are presented in the following parts.

\section{B. Link Utilization}

The link utilization result indicates link such as $L_{16}$ has higher utilization due to whole power system measurement and status are concentrated at control center for its centralized control and monitoring applications as shown in Figure 6. Therefore, when event occurs link utilizations of both $10 \mathrm{mbps}$ and $100 \mathrm{mbps}$ scenarios reach almost $100 \%$. Utilizations of most other links have similar performance as Figure 7. During the steady state utilizations of the link do not reach $100 \%$ in both $10 \mathrm{mbps}$ (around $70 \%$ ) and 100mbps (around $7 \%$ ). However, during the event period, in $10 \mathrm{mbps}$ scenario link utilization reaches almost $100 \%$ but in $100 \mathrm{mbps}$ scenario link utilization reaches around $22 \%$. For some links such as $S S_{4 \_7 \_8 \_9}$ to $S S_{-5 \_6}$ has higher link utilization compared to other links due to bandwidth of this link is shared by more applications. UDP and TCP sychrophasor data configuration scenarios have similar link utilization. In both Figure 6 and Figure 7, it can be found TCP retransmission mechanism leads to boost of utilization.

\section{Link performance for $P O D$}

The input data latency performance for POD application is illustrated in Figure 8

It can be found that due to the congestion link between $S S_{2}$ and $S S_{5}$ during the event period, the latency of synchrophasor data increases dramatically in $10 \mathrm{mbps}$ scenario. However, in $100 \mathrm{mbps}$ scenario, the effect of the burstiness traffic on synchrophasor data is much less. However, in TCP sychrophasor scenario, the report rate is much less than UDP sychrophasor scenario due to congestion control in the data link. 


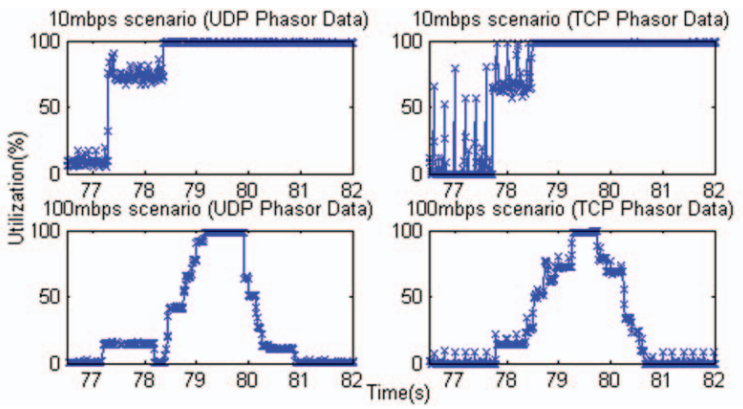

Fig. 6: Link Utilization of link from $S S_{5 \_}$to Control Center.

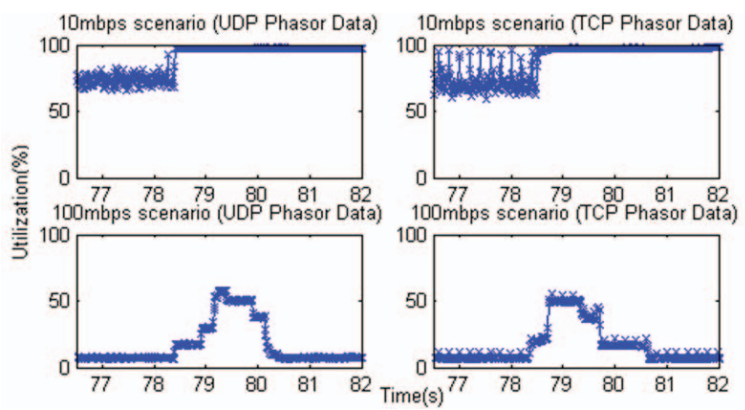

Fig. 7: Link Utilization of link from $S S_{44_{-} 7_{-}{ }_{-} 9}$ to $S S_{5 \_6}$.

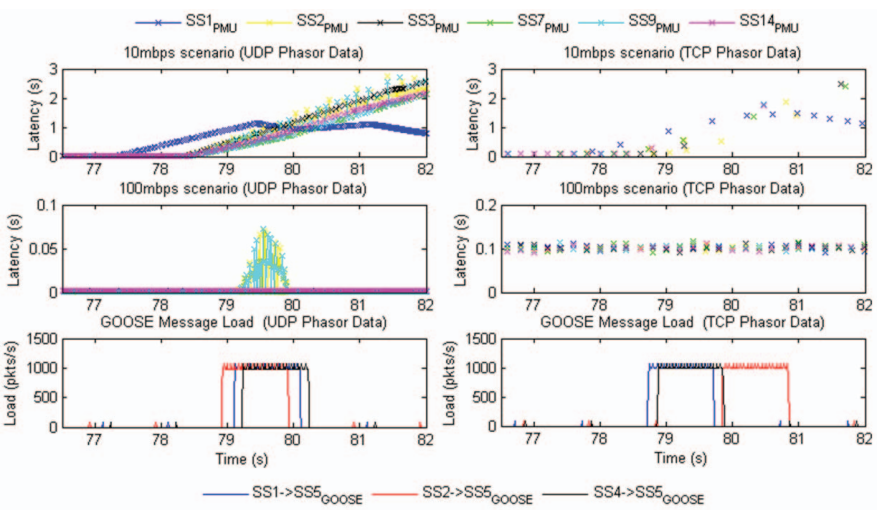

Fig. 8: Input data latency for POD application.

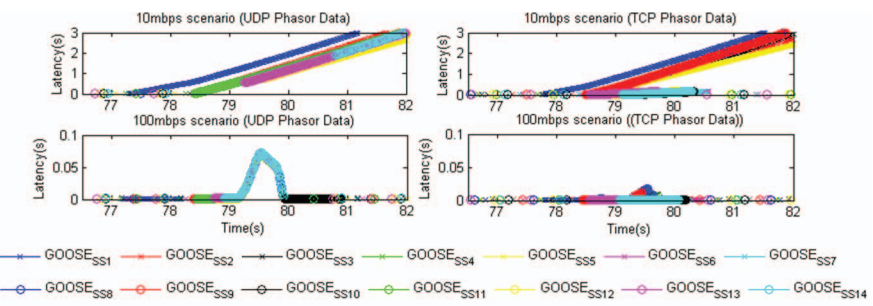

Fig. 9: Input data latency for CRAS application.

\section{Link Performance for CRAS}

The input data latency performance for CRAS is shown in Figure 9.

In both $10 \mathrm{mbps}$ and $100 \mathrm{mbps}$ scenarios, the data acquisition from local substation to control center suffer big latency during the event period due to data link congestion. Since 100mbps

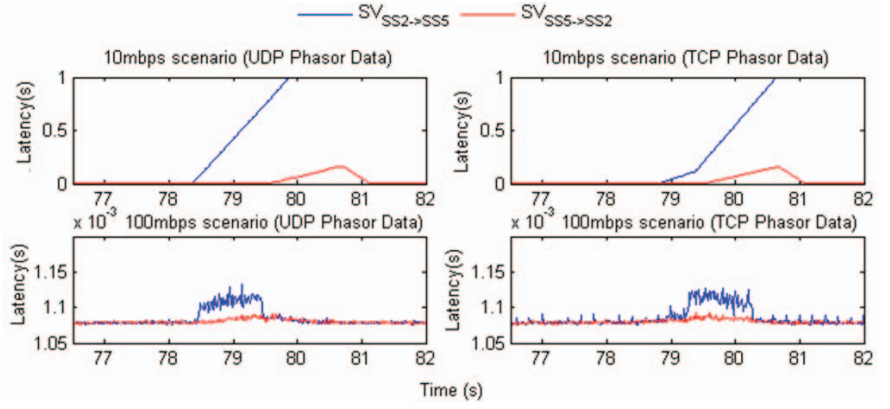

Fig. 10: Input data latency for CDLP application.

link can resolve the accumulated bursty traffic quicker than $10 \mathrm{mbps}$ link. Therefore, the maximum latency in this scenario reaches $0.07 \mathrm{~s}$ and $0.03 \mathrm{~s}$ in UDP and TCP synchrophasor data scenarios separately. However, in $10 \mathrm{mbps}$ scenario, communication system requires more time to digest the accumulated traffic from event period. Therefore, the latency performance is worse. For some data, TCP synchrophasor scenario provides better latency performance compared with UDP sychrophasor scenario thanks to the re-transmission mechanism of TCP.

\section{E. Link performance for online SE}

The input data latency performance for online SE is similar to the input data latency performance for CRAS. Similar to CRAS, 100mbps TCP scenario provides bettern latency performance than $100 \mathrm{mbps}$ UDP scenario. However, in $10 \mathrm{mbps}$ scenarios, both UDP and TCP configuration for phasor data link fail to meet the application requirement.

\section{F. Link Performance for CDLP}

The input data latency performance for CDLP on transmission line between $S S_{2}$ and $S S_{5}$ is shown in Figure 10.

Due to the congestion link between $S S_{2}$ and $S S_{5}$ during the event period, the latency of SV streams exchanged between $S S_{2}$ and $S S_{5}$ increase dramatically in $10 \mathrm{mbps}$ scenarios. However, in 100 mbps scenarios, the effect of the bursty GOOSE traffic on SV streams between substations can be neglected. It also can be found that to the same physical link, effect of bursty GOOSE traffic is direction dependent. The SV stream from $S S_{2}$ to $S S_{5}$ suffers more latency compared with the SV stream from $S S_{5}$ to $S S_{2}$. Since the bandwidth of physical link in direction $S S_{2}$ to $S S_{5}$ is shared other application requires data from $S S_{2}$ to control center.

\section{G. Link Performance for Interlocking}

The input data latency performance for Interlocking is shown in Figure 11.

Similar to Current Differential Line Protection application, data packet for interlocking applications also suffers huge amount of latency in the 10 mbps scenario due to the bursty traffic during the event period. However, the effect of bursty traffic can be neglected in 100 mbps scenario. 
10 mbps scenario (UDP Phasor Data)
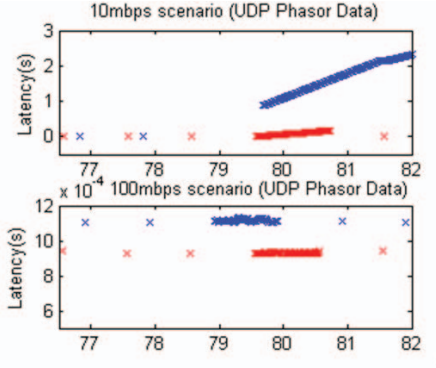

$\longrightarrow$ GOOSE $_{S S 2->S S S}$
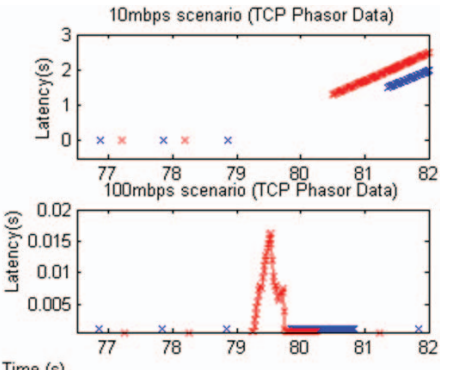

Time (s)

GOOSE $E_{S S S . S S 2}$

Fig. 11: Input data latency for Interlocking application.

\section{H. Result Summary}

From the results presented above, in normal situation, the latency performance in either $10 \mathrm{mbps}$ or $100 \mathrm{mbps}$ scenario fulfills the requirement of all applications regardless TCP or UDP synchrophasor data configuration approach. However, during the event period, bursty traffic can be found in the communication network due to the GOOSE over UDP configuration. Such bursty traffic consumes corresponding network resource dramatically, that leads to the effect on other data link performance. Due to these effects, the requirement of applications cannot be fulfilled under $10 \mathrm{mbps}$ scenario as shown in Table IV. In 100mbps and TCP synchrophasor data configuration scenario, data link performance of POD and interlocking applications fail to fulfill their QoS requirements.

TABLE IV: Application QoS Requirement Fulfillments under different scenarios

\begin{tabular}{|l|l|l|l|l|l|l|l|l|}
\hline \multirow{3}{*}{ Application } & \multicolumn{4}{|l|}{ UDP } & \multicolumn{2}{l|}{ TCP } \\
\cline { 2 - 9 } & \multicolumn{2}{|l|}{ Normal } & \multicolumn{2}{l|}{ Event } & \multicolumn{2}{l|}{ Normal } & \multicolumn{2}{l|}{ Event } \\
\cline { 2 - 9 } & 10 & 100 & 10 & 100 & 10 & 100 & 10 & 100 \\
\hline POD & $\mathbf{Y}$ & $\mathbf{Y}$ & $\mathbf{N}$ & $\mathbf{Y}$ & $\mathbf{Y}$ & $\mathbf{Y}$ & $\mathbf{N}$ & $\mathbf{N}$ \\
\hline CDLP & $\mathbf{Y}$ & $\mathbf{Y}$ & $\mathbf{N}$ & $\mathbf{Y}$ & $\mathbf{Y}$ & $\mathbf{Y}$ & $\mathbf{N}$ & $\mathbf{Y}$ \\
\hline Interlocking & $\mathbf{Y}$ & $\mathbf{Y}$ & $\mathbf{N}$ & $\mathbf{Y}$ & $\mathbf{Y}$ & $\mathbf{Y}$ & $\mathbf{N}$ & $\mathbf{N}$ \\
\hline CRAS & $\mathbf{Y}$ & $\mathbf{Y}$ & $\mathbf{N}$ & $\mathbf{Y}$ & $\mathbf{Y}$ & $\mathbf{Y}$ & $\mathbf{N}$ & $\mathbf{Y}$ \\
\hline Online SE & $\mathbf{Y}$ & $\mathbf{Y}$ & $\mathbf{N}$ & $\mathbf{Y}$ & $\mathbf{Y}$ & $\mathbf{Y}$ & $\mathbf{N}$ & $\mathbf{Y}$ \\
\hline
\end{tabular}

\section{CONCLUSions AND Future ResEARCH}

In this paper, a detailed model of realistic communications for the standard IEEE 14-bus system has been developed. By literature review, control, monitoring, and protection applications have been deployed in the system. The data link configuration and corresponding QoS requirements have been identified. Four scenarios combined different bandwidth and sychrophasor data implementation have been implemented in the simulation. Based on the simulation results, significant effects of bursty traffic from GOOSE distrubance event messages on the delays suffered by steady state periodic traffic have been revealed. $10 \mathrm{mbps}$ data links are inadequate in the face of such bursty event traffic but $100 \mathrm{mbps}$ data link is suffice. However, the results also illustrate that centralized control center architecture has significant scalability issues. Although $100 \mathrm{mbps}$ data link is suffice in this system, such bandwidth might be inadequate when system expanding. Without any strong QoS mechanisms such as GridStat [1] or ATM, the only way to ensure adequate performance is to guarantee low link utilization (i.e., massive overprovisioning of bandwidth). However, it brings high cost of infrastructure and low efficient of communication infrastructure. As continuation of this paper, authors are working on using GridStat to manage these bursty event traffic to ensure the QoS requirements of power system application meanwhile increasing the efficiency of communication infrastructure.

\section{REFERENCES}

[1] D. Bakken, A. Bose, C. Hauser, D. Whitehead, and G. Zweigle, "Smart generation and transmission with coherent, real-time data," Proceedings of the IEEE, vol. 99, no. 6, pp. 928-951, June 2011.

[2] F. Clavel, E. Savary, P. Angays, and A. Vieux-Melchior, "Integration of a new standard: A network simulator of iec 61850 architectures for electrical substations," IEEE Industry Applications Magazine, vol. 21, no. 1 , pp. 41-48, Jan 2015

[3] "IEEE Standard for Synchrophasor Data Transfer for Power Systems," IEEE Std. C37.118.2-2011, 2011.

[4] "Communication networks and systems for power utility automation part 9-2: Specific communication service mapping ( $\mathrm{scsm})$ - sampled values over iso/iec 8802-3," IEC Std. 61850-9-2, 2011.

[5] "Telecontrol equipment and systems - part 5-104: Transmission protocols - network access for iec 60870-5-101 using standard transport profiles," IEC Std. 60870-5-104, 2006.

[6] R. Christie, "Power Systems Test Case Archive," https://www.ee.washington.edu/research/pstca/, 1993, [Online; accessed 10-May-2015].

[7] "Communication networks and systems for power utility automation part 90-1: Use of iec61850 for the communication between substations," IEC Std. 61850-90-1, 2008.

[8] S. Azad, R. Iravani, and J. Tate, "Damping inter-area oscillations based on a model predictive control (mpc) hvdc supplementary controller," Power Systems, IEEE Transactions on, vol. 28, no. 3, pp. 3174-3183, Aug 2013.

[9] B. Chaudhuri, R. Majumder, and B. Pal, "Wide-area measurement-based stabilizing control of power system considering signal transmission delay," IEEE Transactions on Power Systems, vol. 19, no. 4, pp. 19711979, Nov 2004.

[10] J. H. Chow and S. G. Ghiocel, An Adaptive Wide-Area Power System Controller using Synchrophasor Data. Springer, 2012.

[11] A. Bose, "Smart transmission grid applications and their supporting infrastructure," Smart Grid, IEEE Transactions on, vol. 1, no. 1, pp. 11-19, June 2010.

[12] G. Zweigle, "Emerging wide-area power applications with mission critical data delivery requirements," in Smart Grids: Clouds, Communications, Open Source, and Automation, D. Bakken and K. Iniewski, Eds. CRC Press, 2014.

[13] M. Selvan and K. Swarup, "Dynamic topology processing in a radial distribution system," IEE Proceedings-Generation, Transmission and Distribution, vol. 153, no. 2, pp. 155-163, March 2006.

[14] N. Honeth, A. Saleem, K. Zhu, L. Vanfretti, and L. Nordstrom, "Decentralized topology inference of electrical distribution networks," in Innovative Smart Grid Technologies (ISGT), 2012 IEEE PES, Jan 2012, pp. $1-8$.

[15] M. Farrokhabadi and L. Vanfretti, "Phasor-assisted automated topology processing for state estimators," in Electrical Power Energy Conference (EPEC), 2013 IEEE, Aug 2013, pp. 1-8.

[16] K. Jamuna and K. Swarup, "Two stage state estimator with phasor measurements,' in Power Systems, 2009. ICPS '09. International Conference on, Dec 2009, pp. 1-5.

[17] J. Wen, W.-H. Liu, P. Arons, and S. Pandey, "Evolution pathway towards wide area monitoring and protection: A real-world implementation of centralized ras system," Smart Grid, IEEE Transactions on, vol. 5, no. 3, pp. 1506-1513, May 2014. 